\title{
Curcumin inhibits prostate cancer progression by regulating the miR-30a-5p/PCLAF axis
}

\author{
LIANG PAN $^{1 *}$, JIAN SHA $^{1 *}$, WENYAO LIN $^{1}$, YUXIONG WANG $^{1}$, TINGZHANG BIAN $^{1}$ and JIANMING GUO $^{2}$ \\ ${ }^{1}$ Department of Urology, Xuhui Hospital, Zhongshan Hospital Affiliated to Fudan University, Shanghai 200031; \\ ${ }^{2}$ Department of Urology, Zhongshan Hospital Affiliated to Fudan University, Shanghai 200032, P.R. China
}

Received March 18, 2020; Accepted June 4, 2021

DOI: $10.3892 /$ etm.2021.10401

\begin{abstract}
Curcumin has been shown to inhibit the growth of a variety of tumor cells. However, the biological functions of curcumin in prostate cancer (PCa) have not yet fully elucidated. The objective of the present study was to investigate the role of curcumin on the proliferation, migration, invasion and apoptosis of PCa cells and the underlying mechanism. Cell Counting Kit-8 and flow cytometry were used to detect the effects of curcumin at different concentrations on the proliferation and apoptosis of PCa cell lines, PC-3 and DU145. BrdU and Transwell assays, western blotting and reverse transcription-quantitative PCR were used to determine the effect of curcumin on cell proliferation, migration and invasion, apoptosis-related protein expression, and microRNA (miR)-30a-5p and PCNA clamp associated factor (PCLAF) expression, respectively. In addition, bioinformatics analysis and Pearson's correlation test were used to verify the relationship between miR-30a-5p and PCLAF. Curcumin was observed to impede the proliferation, migration and invasion of PCa cells, and promote their apoptosis in a timeand dose-dependent manner. Curcumin enhanced miR-30a-5p expression and inhibited PCLAF expression; furthermore, there was a negative correlation between miR-30a-5p and PCLAF expression in PCa tissues. In addition, transfection of miR-30a-5p inhibitors partially reversed the function of curcumin on cell proliferation, migration, invasion and apoptosis. Overall, curcumin suppressed the malignant biological behaviors of $\mathrm{PCa}$ cells by regulating the miR-30a-5p/PCLAF axis.
\end{abstract}

\section{Introduction}

In 2019, prostate cancer (PCa) was one of the most common malignancies among males worldwide (1). Patients with early

Correspondence to: Dr Jian Sha, Department of Urology, Xuhui Hospital, Zhongshan Hospital Affiliated to Fudan University, 966 Huaihai Middle Road, Xuhui, Shanghai 200031, P.R. China E-mail: author@mail.com

*Contributed equally

Key words: prostate cancer, curcumin, microRNA-30a-5p, PCNA clamp associated factor stages of PCa can be cured by radical surgery or radiotherapy, but the treatment of advanced prostate cancer is still dependent on androgen deprivation therapy (ADT) (2). Although ADT can effectively inhibit the progression of $\mathrm{PCa}$, resistance of PCa cells to ADT can lead to tumor metastasis (3). Therefore, novel therapy strategies are urgently needed in the treatment of PCa.

Curcumin is a polyphenolic compound derived from Curcuma longa L., which is used as perfume in India and herbal medicine in China (4,5). Previous studies indicate that curcumin has anti-inflammatory, anti-oxidative, anti-infective, anti-fibrotic and anti-atherosclerotic effects, and that it exhibits low toxicity $(5,6)$. The anticancer properties of curcumin have attracted increasing attention. Accumulating studies have indicated that curcumin inhibits the occurrence, development, chemoresistance and radioresistance of various malignancies (7-13). For example, curcumin inhibits the proliferation of colon cancer cells by inhibiting the Wnt/ $\beta$-catenin signaling pathway, and can increase the radiosensitivity of PCa cells by inhibiting autophagy $(11,12)$. In addition, curcumin suppresses the activity of JNK signaling in PCa cells, thereby impeding cell proliferation and promoting apoptosis (13). Nevertheless, the mechanism of curcumin in blocking PCa progression has not been clearly clarified.

Certain studies have reported that curcumin can inhibit cancer progression by regulating microRNA (miRNA/miR) expression (7,14-15). For example, in PCa, curcumin inhibits cancer cell proliferation and migration by regulating the miR-143/phosphoglycerate kinase 1 axis (14); curcumin induces the upregulation of miR-145, which in turn inhibits PCa cell proliferation by repressing octamer-binding transcription factor 4 expression (15). Therefore, the present study was designed to uncover the biological functions and specific mechanisms of curcumin/miR-30a-5p/PCNA clamp associated factor (PCLAF) axis in PCa via in vitro assays.

\section{Materials and methods}

Clinical specimens. PCa tissues and the adjacent benign tissues (at 5-cm distance) of 35 patients with $\mathrm{PCa}$ who visited Zhongshan Hospital Affiliated to Fudan University (Shanghai, China) from March 2017 to March 2019 were collected. All of the patients were diagnosed via biopsy and received radical prostatectomy. There were 21 male and 14 female patients. 


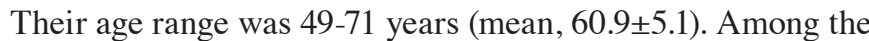
patients enrolled, the Gleason score was $\geq 8$ points in 11 cases and $<8$ points in 24 cases, and the serum prostate-specific antigen levels were $\leq 10 \mathrm{ng} / \mathrm{ml}$ in 19 cases and $>10 \mathrm{ng} / \mathrm{ml}$ in 16 cases. All tissue specimens were surgically removed and quickly frozen in liquid nitrogen. The study was approved by The Ethics Review Board of Zhongshan Hospital Affiliated to Fudan University, and all participants provided written informed consent.

Cell culture, drug treatment and transfection. Both PC-3 and DU145 cells were purchased from the China Center for Type Culture Collection. The miR-30a-5p inhibitor (5'-CUUCCAGUCGAGGAUGUUUACA-3') and non-targeting control (5'-ACAUAGGGCCCAUGCUAACUGC-3') were procured from Guangzhou RiboBio Co., Ltd. PC-3 and DU145 cells were cultured in RPMI-1640 medium (Thermo Fisher Scientific, Inc.) containing 10\% FBS (Thermo Fisher Scientific, Inc), $100 \mu \mathrm{g} / \mathrm{ml}$ penicillin and $100 \mu \mathrm{g} / \mathrm{ml}$ streptomycin (Sigma-Aldrich; Merck KGaA) at $37^{\circ} \mathrm{C}$ in $5 \% \mathrm{CO}_{2}$. When the cells had reached $70-80 \%$ confluence, the miR-30a-5p inhibitor and control were transfected into PC-3 and DU145 cells at $37^{\circ} \mathrm{C}$ for $6 \mathrm{~h}$ at a final concentration of $50 \mu \mathrm{M}$ according to the manufacturer's instructions of Lipofectamine ${ }^{\circledR} 2000$ (Thermo Fisher Science, Inc.). After 24 h, cells were treated with curcumin (cat. no. 239802; Sigma-Aldrich; Merck KGaA) at different concentrations $(10,20,30,40$ or $50 \mu \mathrm{mol} / 1)$ at $37^{\circ} \mathrm{C}$ for 12,24 or $48 \mathrm{~h}$. After that, cells were harvested for subsequent experiments.

Cell Counting Kit-8 (CCK-8) assay. PC-3 and DU145 cells in the logarithmic growth phase were seeded in a 96-well cell plate $\left(2 \times 10^{3}\right.$ cells/well). Subsequently, the cells were randomly divided into six groups with different concentrations of curcumin: i) Control group ( $0 \mu \mathrm{mol} / \mathrm{l})$; ii) $10 \mu \mathrm{mol} / 1$ curcumin group; iii) $20 \mu \mathrm{mol} / 1$ curcumin group; iv) $30 \mu \mathrm{mol} / 1$ curcumin group; v) $40 \mu \mathrm{mol} / 1$ curcumin group; and vi) $50 \mu \mathrm{mol} / 1$ curcumin group. CCK-8 solution (10 $\mu \mathrm{l}$; Beyotime Institute of Biotechnology) was supplemented to each well at 12, 24 and $48 \mathrm{~h}$, and then the cells were incubated at $37^{\circ} \mathrm{C}$ for a further $4 \mathrm{~h}$. After that, the absorbance value of each well was detected by a microplate reader (GENios FL Fluorescence Microplate Reader FI TRF; Tecan Group, Ltd.) at a wavelength of $450 \mathrm{~nm}$, and the average absorbance value of three wells were used to evaluate the viability of cells.

Flow cytometry analysis. The Annexin V-FITC/propidium iodide (PI) double staining method was used to analyze the apoptosis of PCa cells in this study. A total of $1 \times 10^{6}$ PC-3 or DU145 cells from each group were harvested and resuspended using $100 \mu \mathrm{l} 1 \mathrm{X}$ binding buffer (BSL Bioservice), followed by the addition of $5 \mu \mathrm{l}$ Annexin V-FITC solution and $5 \mu \mathrm{l}$ PI solution (both from Beyotime Institute of Biotechnology), mixed thoroughly and incubated in the dark at room temperature for 15 min. Subsequently, the apoptosis rate was detected using a flow cytometer (BD Biosciences) within $1 \mathrm{~h}$ and the data were analyzed using FlowJo v10.07 software (FlowJo, LLC).

Reverse transcription-quantitative PCR (RT-qPCR). PC-3 and DU145 cells treated with $30 \mu \mathrm{mol} / 1$ curcumin for $24 \mathrm{~h}$ were collected. Total cellular RNA was extracted using TRIzol ${ }^{\circledR}$ reagent (Invitrogen; Thermo Fisher Scientific, Inc.). RNA concentration and purity were measured on the NanoDrop 2000 (NanoDrop Technologies; Thermo Fisher Scientific, Inc.) using $1 \mu \mathrm{l}$ of RNA. After the concentration and purity of RNA were determined, total RNA was reverse transcribed into cDNA using a RevertAid First Strand cDNA Synthesis Kit ( $1 \mathrm{~h}$ at $37^{\circ} \mathrm{C}$; Thermo Fisher Scientific, Inc.). RT-qPCR was conducted using a CFX96 quantitative PCR system (Bio-Rad Laboratories, Inc.) with an SYBR ${ }^{\circledR}$ Green Premix Ex Taq II kit (Takara Biotechnology Co., Ltd.) in accordance with the manufacturer's instructions. The following temperature protocol was used for reverse transcription: $37^{\circ} \mathrm{C}$ For $2 \mathrm{~min}$, $23^{\circ} \mathrm{C}$ for $10 \mathrm{~min}, 55^{\circ} \mathrm{C}$ for $10 \mathrm{~min}$ and $85^{\circ} \mathrm{C}$ for $10 \mathrm{~min}$. U6 was identified as the internal reference for miR-30a-5p, and $\beta$-actin was used as the internal reference for PCLAF. The levels of miR-30a-5p and PCLAF mRNA expression were calculated using the $2^{-\Delta \Delta \mathrm{Cq}}$ method (16). The primer sequences were shown in Table I.

BrdU experiment. PC-3 and DU145 cells were prepared into a single-cell suspension and inoculated in a 96-well plate ( $1 \times 10^{4}$ cells/well). The cells were then divided into control- and curcumin-treated groups. After being treated with $30 \mu \mathrm{mol} / \mathrm{l}$ curcumin for $24 \mathrm{~h}$, BrdU solution (Beyotime Institute of Biotechnology) was added at a final concentration of $30 \mu \mathrm{mol} / 1$ and then the cells were incubated at $37^{\circ} \mathrm{C}$ for $8 \mathrm{~h}$. The cells were washed with PBS three times. Subsequently, the cells were fixed with $4 \%$ paraformaldehyde at room temperature for $30 \mathrm{~min}$ and rinsed with PBS for three times again. A total of $2 \mathrm{~mol} / 1 \mathrm{HCl}$ was added to denature the DNA for $30 \mathrm{~min}$ at $37^{\circ} \mathrm{C}$ and then cells were immersed in PBS containing $0.1 \%$ Tween-20 for $30 \mathrm{~min}$. After that, non-specific antigens were blocked with 3\% BSA (Sigma-Aldrich; Merck KGaA) for $1 \mathrm{~h}$ at room temperature. Following which, mouse anti-BrdU antibody (1:1400; cat. no. 5292S; Cell Signaling Technology, Inc.) was incubated with the cells for $2 \mathrm{~h}$ at room temperature. The cells were rinsed for three times with PBS, and the secondary antibody incubated with the cells for $1 \mathrm{~h}$ at room temperature. Subsequently, DAPI staining solution was used to stain the nuclei at $37^{\circ} \mathrm{C}$ for $30 \mathrm{~min}$. The cells were observed and images captured under a fluorescence microscope (magnification, x400; Olympus Corporation). Overall, five visual fields were observed under fluorescence microscope for each sample. Cell proliferation rate $=$ the number of BrdU staining positive cells/total DAPI staining positive cells x100\%.

Scratch assay. Wound healing was used to evaluate PCa cell migration. PC-3 and DU145 cells were seeded into six-well plates at a density of $5 \times 10^{5}$ cells/well. After the cells were cultured to $100 \%$ confluence and serum starved for $24 \mathrm{~h}$, a sterile $200-\mu 1$ pipette tip was used to scratch the cells at the center of the well. The cells were washed with PBS to remove the cell debris, and the cells were cultured in serum-free medium. Closure of the wound was observed under an inverted fluorescence microscope (magnification, x400; Olympus Corporation) at 0 and $24 \mathrm{~h}$ after scratching.

Transwell assay. Cells in each group were trypsinized, harvested and resuspended in serum-free medium to adjust the 
Table I. Primer sequences used for reverse transcriptionquantitative PCR.

Name Primer sequences, 5'-3'

miR-30a-5p forward GGGCCTGTAAACATCCTCG

miR-30a-5p reverse GAATACCTCGGACCCTGC

PCLAF forward

ATGGTGCGGACTAAAGCAGAC

PCLAF reverse CCTCGATGAAACTGATGTCGAAT

U6 forward GCTTCGGCAGCACATATACTAAAAT

U6 reverse CGCTTCACGAATTTGCGTGTCAT

$\beta$-actin forward TCCCTCAAGATTGCTAGCAA

$\beta$-actin reverse AGATCCACAACGGATACATT

miR, microRNA; PCLAF, PCNA clamp associated factor.

cell concentration to $5 \times 10^{5}$ cells $/ \mathrm{ml}$. To evaluate the migration of cells, a Transwell system ( $8-\mu \mathrm{m}$ pore-size; Corning, Inc.) was used. A total of $200 \mu \mathrm{l}$ cell suspension was added to the upper chamber, $600 \mu 1$ RPMI-1640 medium containing 10\% FBS (both from Thermo Fisher Scientific, Inc.) was added to the lower chamber and the cells were cultured in an incubator for $24 \mathrm{~h}$. Subsequently, the cells in the upper chamber were carefully wiped off. The migrated cells were fixed with formaldehyde at $37^{\circ} \mathrm{C}$ for $15 \mathrm{~min}$, and then stained with crystal violet solution at $37^{\circ} \mathrm{C}$ for $30 \mathrm{~min}$. After being rinsed with PBS, the cells were dried and observed under an inverted fluorescence microscope (magnification, x400). Overall, five visual fields were observed under fluorescence microscope for each sample. To test cell invasion, the membrane of the Transwell system was coated with Matrigel for $1 \mathrm{~h}$ at $37^{\circ} \mathrm{C}$ (BD Biosciences) before the experiment, and the other steps were as the same as the migration assay.

Western blotting. PC-3 and DU145 cells were treated with $30 \mu \mathrm{mol} / 1$ curcumin for $24 \mathrm{~h}$, and then the protease inhibitor-containing RIPA buffer (Beyotime Institute of Biotechnology) was added to lyse the cells on ice for $40 \mathrm{~min}$. Then, the lysate was centrifuged at $1,500 \mathrm{x}$ g for $15 \mathrm{~min}$ to obtain the supernatant. Protein concentration determination was performed using the BCA protein determination assay and $30 \mu \mathrm{g}$ of protein was loaded per lane. Thereafter, the protein samples were loaded, $10 \%$ SDS-PAGE was performed and then the proteins were electrically transferred onto the PVDF membrane (EMD Millipore). After that, 5\% skim-milk was used to block the non-specific antigens at room temperature. Next, primary antibodies were added to incubate the membrane at $4^{\circ} \mathrm{C}$ overnight, and then the membrane was subsequently incubated with horseradish peroxidase-conjugated secondary antibody for $1 \mathrm{~h}$ at room temperature. Then, ECL chemiluminescent solution (Beyotime Institute of Biotechnology) was used to visualize the bands and the bands were quantified using ImageJ software (v1.52; National Institutes of Health). The antibodies used in this study were obtained from Abcam: Anti-PCLAF Antibody (cat. no. ab226255; 1:1,000), anti-Bax antibody (cat. no. ab32503; 1:1,000), anti-Bcl-2 antibody (cat. no. ab185002; 1:1,000), anti-caspase-3 antibody (cat. no. ab13847, 1:1,000), anti-cleaved caspase-3 antibody (cat. no. ab2302; $1: 500$ ), anti- $\beta$-actin antibody (cat. no. ab8227; 1:500) and horseradish peroxidase-conjugated goat anti-rabbit secondary antibody (cat. no. ab205718; 1:1,000).

Bioinformatics analysis. The Gene Expression Profiling Interactive Analysis (GEPIA) database (http://gepia.cancer-pku. $\mathrm{cn} /$ ) was used to analyze PCLAF expression in PCa samples. The StarBase v3.0 database (http://starbase.sysu.edu.cn/) was used to analyze the correlation between miR-30a-5p expression and PCLAF expression in PCa samples. Cut-off values were as follows: $\mid \log 2 \mathrm{FCl}$ Cut-off $=1$, P-value cut-off $=0.01$.

Statistical analysis. All data are expressed as mean \pm standard deviation (unless otherwise shown). SPSS v22.0 (IBM Corp.) and GraphPad Prism 8.0 (Version X; GraphPad Software, Inc.) were applied for statistical analysis. All experiments were performed in triplicate. Whether the data were normally distributed was examined using a one-sample Kolmogorov-Smirnov test. For normally distributed data, an independent sample t-test was used to make the comparison between two groups. One-way ANOVA was used to compare three or more groups. If there was a significant difference, Student-Neuman-Keuls or Tukey's tests were used to determine significant differences between two groups. Correlation was analyzed using Pearson correlation coefficient. $\mathrm{P}<0.05$ was considered to indicate a statistically significant difference.

\section{Results}

Function of curcumin on the viability and apoptosis of $\mathrm{PCa}$ cells. PCa cell lines PC-3 and DU145 cells were treated with different concentrations of curcumin $(0,10,20,30$, 40 and $50 \mu \mathrm{mol} / \mathrm{l}$ ), and a CCK-8 assay was employed to detect cell viability at 12,24 and $48 \mathrm{~h}$ after treatment. As shown in Fig. 1A, compared with the untreated group, curcumin inhibited the viability of PCa in concentration- and time-dependent manners. As seen in Figs. 1B and S1, after being treated with curcumin for $24 \mathrm{~h}$, the apoptosis rate of PCa cells gradually increased with increasing concentrations of curcumin. Moreover, when PCa cells were treated with $30 \mu \mathrm{mol} / 1$ curcumin, the apoptotic rate gradually increased with the treatment time (Figs. 1C and S1). These observations suggested that curcumin can inhibit cell viability and promote apoptosis.

Curcumin inhibits the proliferation, migration and invasion of PCa cells and promotes apoptosis. To further examine the role of curcumin on $\mathrm{PCa}$ cell proliferation, migration and invasion, BrdU, wound healing and Transwell assays were performed. As shown in Fig. 2A-C, after PC-3 and DU145 cells were treated with $30 \mu \mathrm{mol} / 1$ curcumin for $24 \mathrm{~h}$, cell proliferation, migration and invasion were significantly reduced compared with the control untreated group. Western blotting was used to detect the expression of apoptosis-related proteins. As shown in Fig. 2D, after treatment with curcumin, the expression levels of Bax and cleaved caspase- 3 were increased in both PC-3 cells and DU145 cells, while the expression levels of $\mathrm{Bcl}-2$ and caspase-3 were decreased. These results further indicated that curcumin exhibited inhibitory effects on PCa cells. 

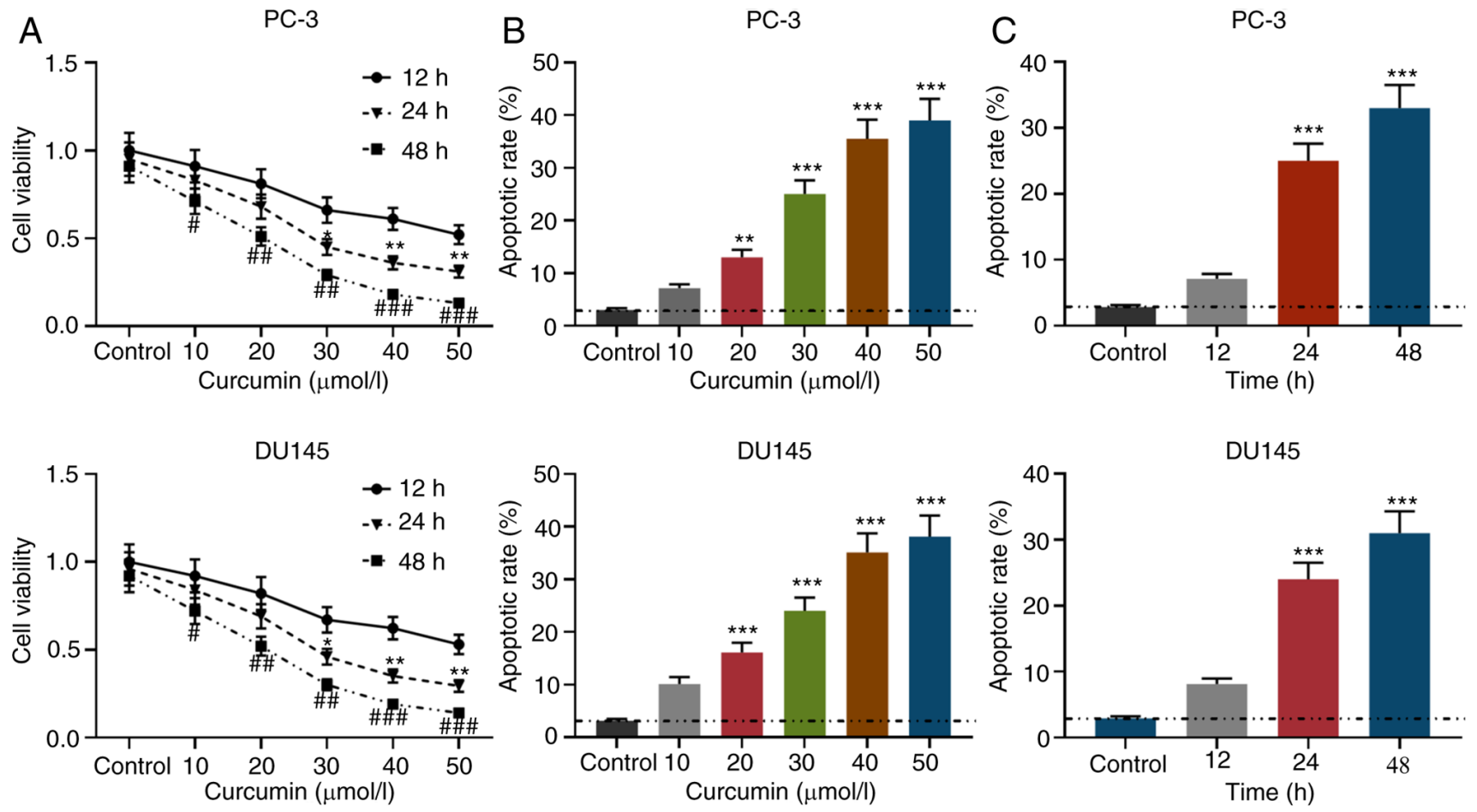

Figure 1. Curcumin inhibits PCa cell viability and promotes apoptosis. (A) Cell Counting Kit- 8 was used to detect the viability of PCa cells treated with different concentrations of curcumin for different times. Flow cytometry was used to detect the apoptosis rates of PCa cells (B) treated with $0-50 \mu$ mol/ 1 of curcumin for $24 \mathrm{~h}$ and $(\mathrm{C})$ treated with $30 \mu \mathrm{mol} / 1$ curcumin for different times. ${ }^{*} \mathrm{P}<0.05,{ }^{* *} \mathrm{P}<0.01,{ }^{* * * *} \mathrm{P}<0.001$ vs. $12 \mathrm{~h}$ or Control; ${ }^{\# P} \mathrm{P}<0.05$, ${ }^{\# \#} \mathrm{P}<0.01,{ }^{\# \# "} \mathrm{P}<0.001$ vs. 24 h. PCa, prostate cancer.

Curcumin suppresses PCLAF expression by upregulating miR-30a-5p in PCa cells. miR-30a-5p exerts a tumor-suppressive effect in colorectal cancer and melanoma by inhibiting cell proliferation, metastasis, differentiation and the cell cycle (17-19). A recent study reported that miR-30a-5p has low expression levels in PCa, and downregulation of miR-30a-5p promotes the progression of $\mathrm{PCa}$ by targeting PCLAF (20). After PC-3 and DU145 cells were treated with $30 \mu \mathrm{mol} / 1$ curcumin for $24 \mathrm{~h}$, the cells were collected and the levels of PCLAF and miR-30a-5p expression in PC-3 and DU145 cells were detected via RT-qPCR and western blotting. The results showed that in comparison with the untreated group, miR-30a-5p expression was significantly increased in PCa cells, while PCLAF expression was decreased (Fig. 3A and B).

Through analyzing the data from GEPIA, it was demonstrated that PCLAF expression was upregulated in $\mathrm{PCa}$ samples compared with normal tissues adjacent to $\mathrm{PCa}$ cancer tissues (Fig. 3C). Further analysis of the data from the StarBase database found that in prostate adenocarcinoma samples, miR-30a-5p expression and PCLAF expression were significant ( $r=-0.215$; Fig. 3D). Levels of miR-30a-5p and PCLAF mRNA expression were also detected in the 35 pairs of $\mathrm{PCa}$ tissues/adjacent tissues collected from patients; miR-30a-5p was downregulated in PCa tissues, while PCLAF was upregulated (Fig. S2A and B). Additionally, the expression levels of miR-30a-5p and PCLAF were negatively correlated in PCa tissues (Fig. 3E). Considering that PCLAF is a target gene of miR-30a-5p (20), it was concluded that curcumin could inhibit the expression of PCLAF by promoting the expression of miR-30a-5p.
Curcumin inhibits the malignant biological behaviors of $P C a$ cells partly by regulating $m i R-30 a-5 p$. To investigate whether curcumin affected PCa cells through regulating miR-30a-5p and PCLAF, before PCa cells were treated with $30 \mu \mathrm{mol} / 1$ curcumin for $24 \mathrm{~h}, \mathrm{miR}-30 \mathrm{a}-5 \mathrm{p}$ inhibitors were transfected into PCa cells (Fig. S2C). As shown in Fig. 4A, the transfection of miR-30a-5p inhibitors partially reversed the inhibitory effect of curcumin on the expression of PCLAF in both PCa cell lines. Subsequently, through the BrdU, wound healing and Transwell assays, it was found that curcumin significantly suppressed PCa cell proliferation, migration and invasion, while miR-30a-5p inhibitors partially reversed these effects (Figs. 4B-D and S1). Additionally, transfection of miR-30a-5p inhibitors partially reversed the effects of curcumin on the expression of $\mathrm{Bax}, \mathrm{Bcl} 2$, cleaved caspase- 3 and caspase-3 (Fig. 4E). These results further validated that the miR-30a-5p/PCLAF axis was a significant downstream effector of curcumin.

\section{Discussion}

A number of studies have shown that curcumin exerts significant anticancer effects in diverse malignancies, inhibiting cancer cell proliferation and metastasis, promoting apoptosis, and increasing chemosensitivity and radiosensitivity (21-24). Curcumin suppresses the proliferation, migration and invasion of PCa cells by regulating multiple signaling pathways. For example, it inhibits the expression of membrane type 1-MMP and MMP2 in PCa cells, and suppresses the viability and metastasis of cancer cells via the Notch-1 signaling pathway (21). A similar study reports that curcumin induces PCa apoptosis and 
A

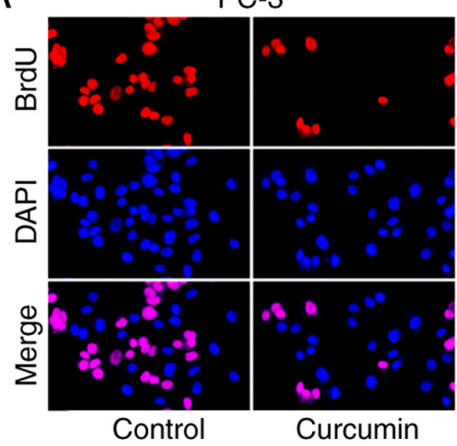

B

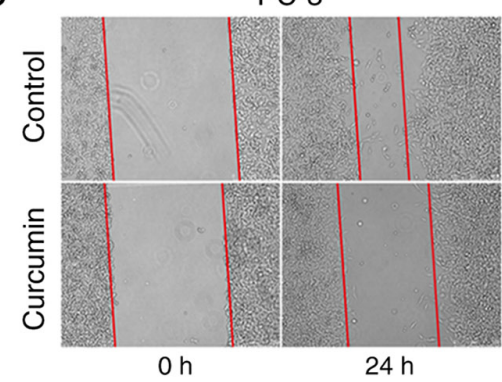

DU145

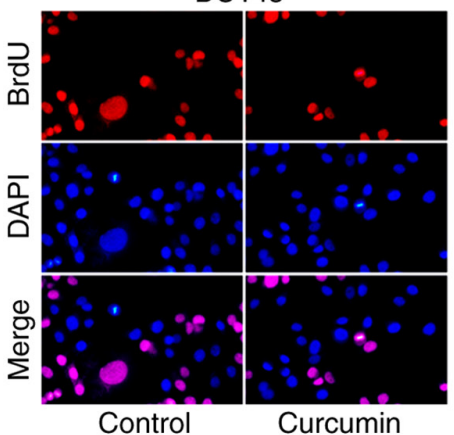

PC-3

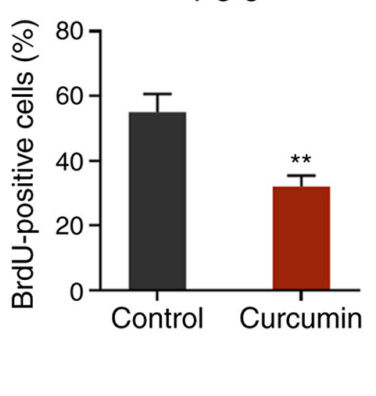

DU145

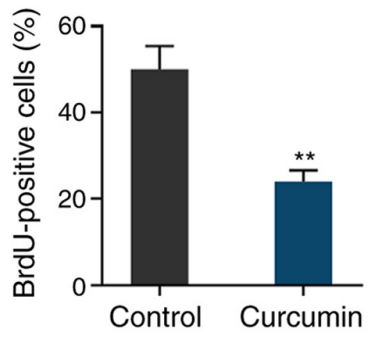

C

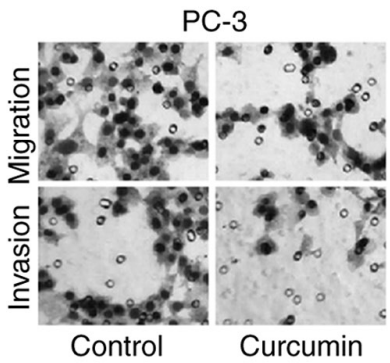

D

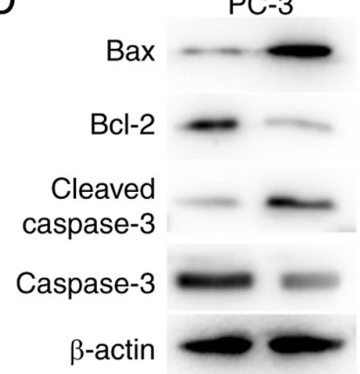

Control Curcumin

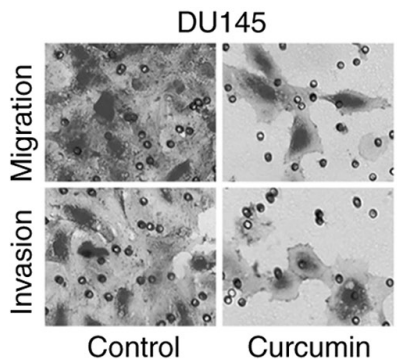

DU145

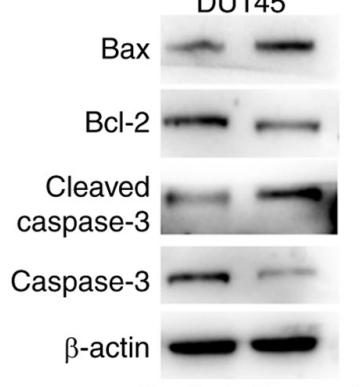

Control Curcumin
DU145
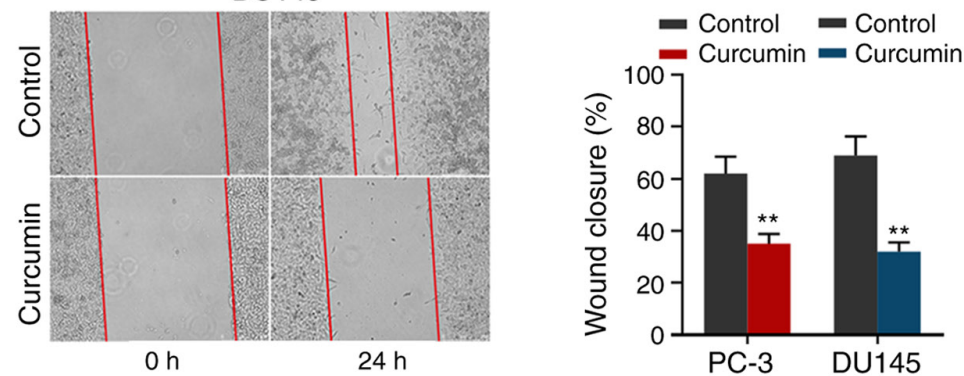

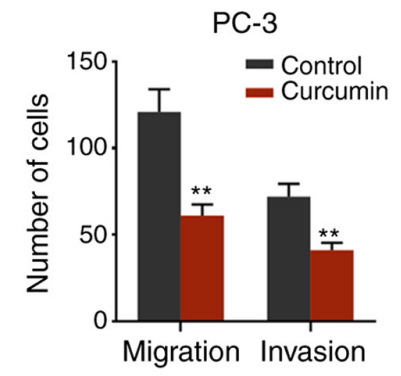

$\mathrm{PC}-3$

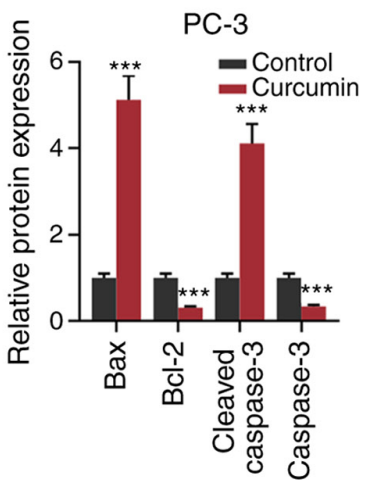

DU145
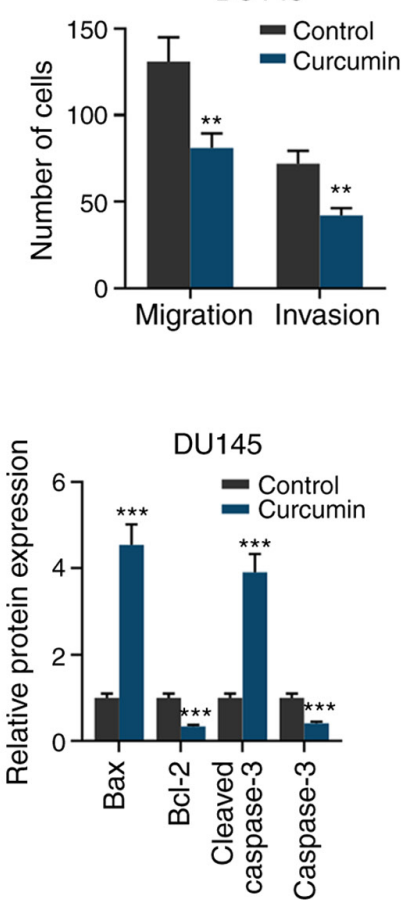

Figure 2. Curcumin inhibits PCa cell proliferation, migration and invasion, and regulates apoptosis-related proteins. (A) BrdU assay was used to detect the proliferation of PCa cells treated with $30 \mu \mathrm{mol} / 1$ curcumin for $24 \mathrm{~h}$ (magnification, $\mathrm{x} 400)$. (B) Wound healing (magnification, $\mathrm{x} 400)$ and (C) Transwell assays were used to detect the of migration and invasion of PCa cells treated with $30 \mu \mathrm{mol} / 1$ curcumin for $24 \mathrm{~h}$ (magnification, $\mathrm{x} 400$ ). (D) Western blotting was used to detect the effect of curcumin on the expression of apoptosis-related proteins. ${ }^{* *} \mathrm{P}<0.01,{ }^{* * *} \mathrm{P}<0.001 \mathrm{vs}$. Control. $\mathrm{PCa}$, prostate cancer.

G0/G1 arrest by inhibiting Notch signaling (22). In addition, curcumin represses the monoamine oxidase A/mTOR/hypoxia inducible factor- $1 \alpha$ signaling pathway, impedes the production of reactive oxygen species, inhibits the expression levels of C-X-C chemokine receptor 4 and IL-6 receptors, and suppresses the epithelial-mesenchymal transition of PCa cells mediated by cancer-associated fibroblasts (23). Moreover, curcumin inhibits nuclear $\beta$-catenin transcription activity in PCa cells by activating polycystin-1, thereby suppressing the proliferation, colony formation and motility of cancer cells (24). In the present study, it was observed that curcumin could inhibit PCa cell proliferation, migration and invasion, and promote apoptosis in a concentration- and time- dependent manner. These experiments indicated that curcumin exhibited tumor-suppressive properties in $\mathrm{PCa}$, which is consistent with previous reports. 
A

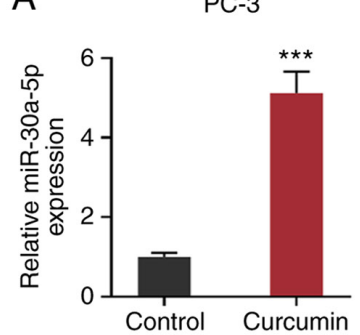

C

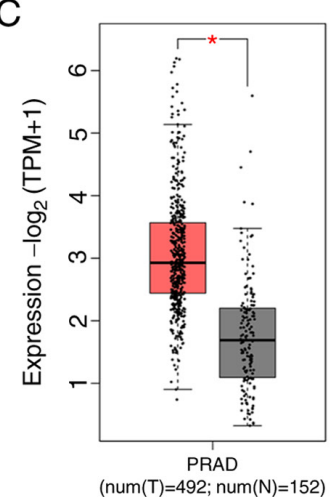

DU145

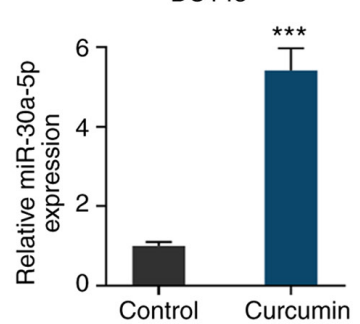

D

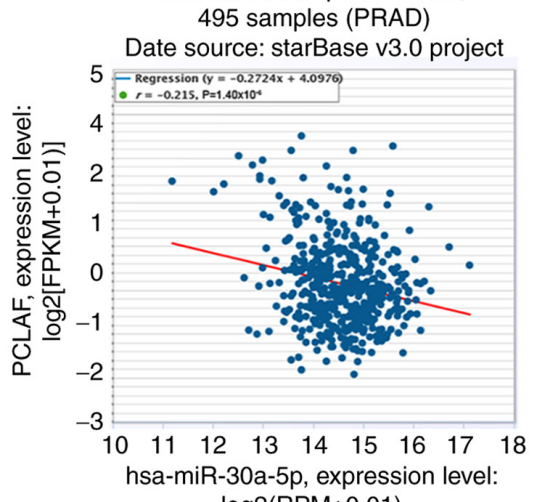

PC-3

B

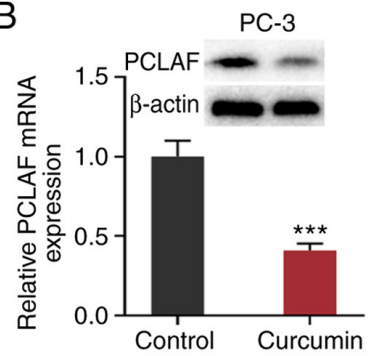

$\mathrm{E}$

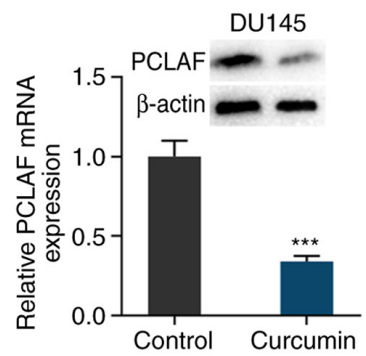

$\log 2(\mathrm{RPM}+0.01)$

Figure 3. Curcumin promotes miR-30a-5p expression and inhibits PCLAF expression. (A) RT-qPCR was employed to detect the effect of curcumin on miR-30a-5p expression. (B) RT-qPCR (left) and western blotting (right) were used to detect the effect of curcumin on PCLAF mRNA and protein expression. (C) PCLAF expression in PCa samples was analyzed using the Gene Expression Profiling Interactive Analysis database. (D) StarBase database was used to analyze the correlation between miR-30a-5p expression and PCLAF mRNA expression in PCa samples. (E) miR-30a-5p expression and PCLAF mRNA expression in 35 patients with PCa were detected using RT-qPCR and their correlation was analyzed. ${ }^{*} \mathrm{P}<0.05$ vs. normal tissue. ${ }^{* * *} \mathrm{P}<0.001$ vs. Control. miR, microRNA; RT-qPCR, reverse transcription-quantitative PCR; PCLAF, PCNA clamp associated factor; PCa, prostate cancer; PRAD, prostate adenocarcinoma; N, normal; T, tumor.

A
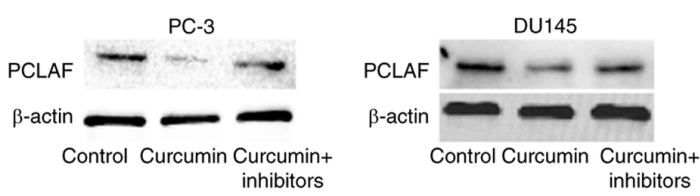

$\mathrm{B}$

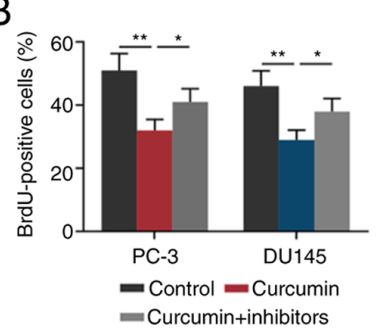

$\mathrm{E}$

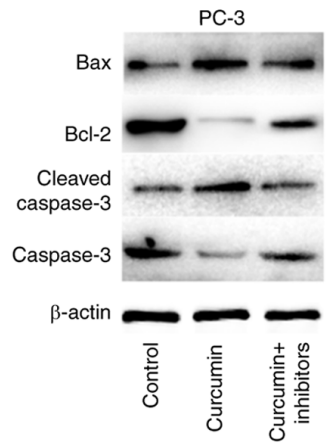

C
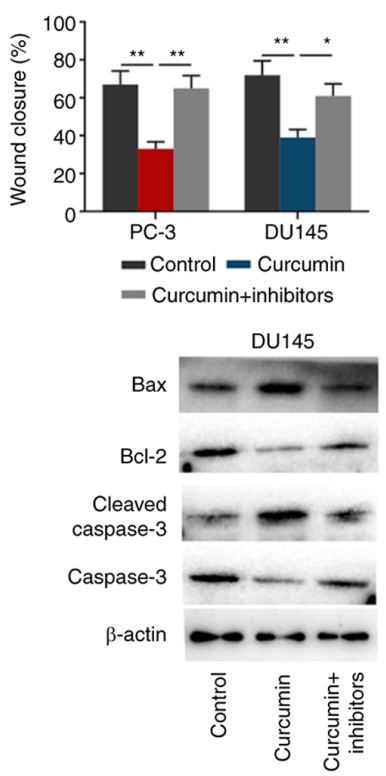

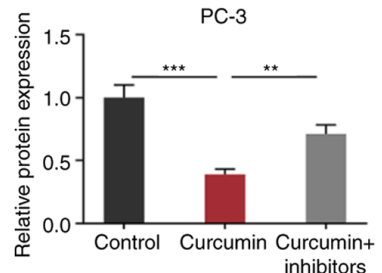

$\mathrm{D}$
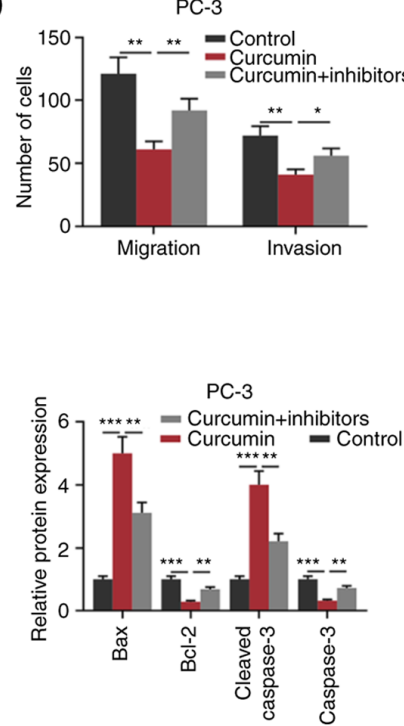

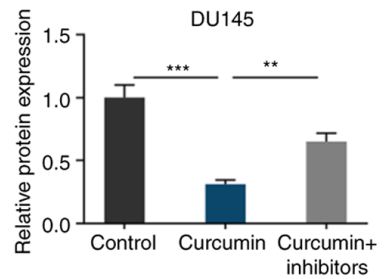

inhibitors
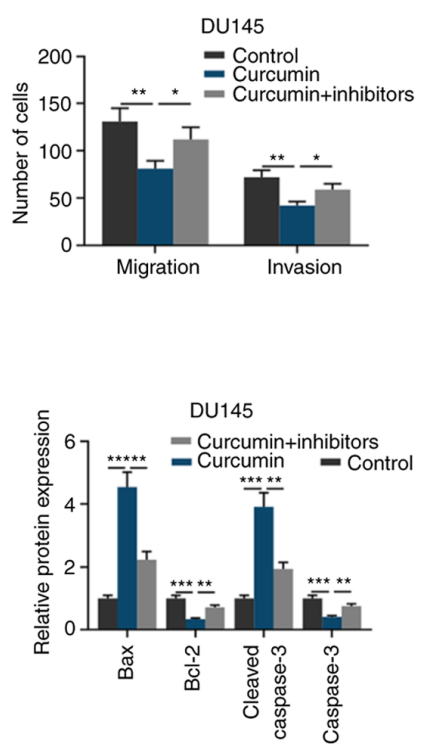

Figure 4. Curcumin inhibits the malignant phenotypes of PCa cells via miR-30a-5p. (A) After transfection of miR-30a-5p inhibitors and curcumin treatment, PCLAF expression in PC-3 cells and DU145 cells was detected via western blotting. (B) BrdU assay was used to detect the proliferation of PCa cells. (C) Wound healing and (D) Transwell assays were used to detect the migration and invasion of PCa cells. (E) Effects of curcumin and miR-30a-5p inhibitors on the expression of apoptosis-related proteins were detected via western blotting. ${ }^{*} \mathrm{P}<0.05,{ }^{* *} \mathrm{P}<0.01,{ }^{* * * *} \mathrm{P}<0.001$. PCa, prostate cancer; miR, microRNA; PCLAF, PCNA clamp associated factor. 
An increasing number of studies have shown that miRNAs have notable effects on cell proliferation, apoptosis and differentiation (25-28). A number of studies report that miRNAs are implicated in carcinogenesis and cancer progression. For example, miR-9-5p can suppress the proliferation of glioma cells by downregulating forkhead box P2 expression (28). In $\mathrm{PCa}, \mathrm{miR}-381$ suppresses the proliferation and invasion of $\mathrm{PCa}$ cells by downregulating androgen receptor expression (29). Curcumin can exert tumor-suppressive effects by regulating the expression of a diverse number of miRs (12,30-33). For example, in laryngeal squamous cell carcinoma, curcumin inhibits the activity of the PI3K/Akt/mTOR signaling pathway by upregulating miR-145 expression, thereby inhibiting the proliferation, cell cycle procession and metastasis of cancer cells (32). In nasopharyngeal carcinoma, curcumin suppresses cancer cell proliferation and metastasis by regulating the miR-7/Skp2/p21 axis (33). In PCa, curcumin represses autophagy through the miR-143/ATG2B axis, thereby increasing the radiosensitivity of cancer cells (12).

The present study observed that miR-30a-5p expression was induced by curcumin treatment in both PC-3 and DU-145 cells. Notably, after PCa cells were transfected with miR-30a-5p inhibitors, the antitumor activity of curcumin was attenuated. These results suggested that miR-30a-5p was a downstream effector of curcumin, and the function of curcumin in inhibiting PCa progression was partly dependent on miR-30a-5p.

miR-30a-5p is well known as a tumor suppressor (34-36). In breast cancer, miR-30a-5p targets the regulation of lactate dehydrogenase A to inhibit the Warburg effect, and impedes the proliferation and metastasis of cancer cells (37). In non-small cell lung cancer, miR-30a-5p downregulates CD73 expression and inhibits proliferation, metastasis and epithelial-mesenchymal transition by regulating the EGF signaling pathway (38). In PCa, miR-30a-5p is also significantly downregulated in cancer tissues, and it suppresses the proliferation of PCa cells by inhibiting PCLAF (20). PCLAF, also known as KIAA0101, is an upregulated oncogene in a variety of tumors (39). Previous studies have shown that PCLAF plays a vital role in cell proliferation, cell survival, DNA repair and tumorigenesis (40-42). For example, PCLAF-knockdown suppresses cancer cell proliferation and cell cycle progression by promoting the formation of $\mathrm{p} 53 / \mathrm{Sp} 1$ complex in breast cancer (41). miR-429 inhibits the progression of epithelial ovarian cancer by targeting PCLAF (42). The present study demonstrated that PCLAF was highly expressed in $\mathrm{PCa}$ samples and that miR-30a-5p expression was negatively associated with PCLAF expression in PCa samples. These data further evidenced the regulatory effect of miR-30a-5p on PCLAF, which is consistent with previous reports (20). Moreover, curcumin treatment downregulated PCLAF expression in both PC-3 and DU145 cell lines. Collectively, these data showed that curcumin could inhibit PCLAF to block the progression of PCa by inducing the expression of miR-30a-5p. However, the weak correlation between miR-30a-5p and PCLAF predicted by bioinformatics in the present study is limitations the conclusions of this study.

In summary, curcumin inhibited the proliferation and metastasis, and promoted the apoptosis of PCa cells by regulating the miR-30a-5p/PCLAF axis. The present study provides novel insights into the mechanism of curcumin's tumor-suppressive functions. Notably, that curcumin may repress the malignant phenotypes of $\mathrm{PCa}$ cells by regulating other miRNAs and proteins, which remains to be clarified in future studies.

\section{Acknowledgements}

Not applicable.

\section{Funding}

The present study was supported by a grant from The Department of Urology, Xuhui Hospital, Zhongshan Hospital Affiliated to Fudan University (grant no. 2017XHYY-05).

\section{Availability of data and materials}

The datasets used and/or analyzed during the current study are available from the corresponding author on reasonable request.

\section{Authors' contributions}

LP, JS and JG conceived and designed the experiments. LP, WL and TB performed the experiments. YW and WL performed the statistical analysis. LP, JS and TB drafted the manuscript. LP, JS and JG confirm the authenticity of all the raw data. All authors have read and approved the final manuscript.

\section{Ethics approval and consent to participate}

The present study was approved by The Ethics Review Board of Zhongshan Hospital Affiliated to Fudan University and all participants provided written informed consent.

\section{Patient consent for publication}

Not applicable.

\section{Competing interests}

The authors declare that they have no competing interests.

\section{References}

1. Guo Z, He C, Yang F, Qin L, Lu X and Wu J: Long non-coding RNA-NEAT1, a sponge for miR-98-5p, promotes expression of oncogene HMGA2 in prostate cancer. Biosci Rep: Sep 24, 2019 (Epub ahead of print). doi: 10.1042/BSR20190635.

2. Song Z, Zhuo Z, Ma Z, Hou C, Chen G and Xu G: Hsa Circ_0001206 is downregulated and inhibits cell proliferation, migration and invasion in prostate cancer. Artif Cells Nanomed Biotechnol 47: 2449-2464, 2019.

3. Dart DA, Koushyar S, Lanning BE and Jiang W: MiR-221 is specifically elevated in PC3 cells and its deletion reduces adhesion, motility and growth. Anticancer Res 39: 5311-5327, 2019.

4. Li H, Yue L, Xu H, Li N, Li J, Zhang Z and Zhao RC: Curcumin suppresses osteogenesis by inducing miR-126a-3p and subsequently suppressing the WNT/LRP6 pathway. Aging (Albany NY) 11: 6983-6998, 2019.

5. Mou S, Zhou Z, He Y, Liu F and Gong L: Curcumin inhibits cell proliferation and promotes apoptosis of laryngeal cancer cells through Bcl-2 and PI3K/Akt, and by upregulating miR-15a. Oncol Lett 14: 4937-4942, 2017. 
6. Zhan JW, Jiao DM, Wang Y, Song J, Wu JH, Wu LJ, Chen QY and Ma SL: Integrated microRNA and gene expression profiling reveals the crucial miRNAs in curcumin anti-lung cancer cell invasion. Thorac Cancer 8: 461-470, 2017.

7. Sun C, Zhang S, Liu C and Liu X: Curcumin promoted miR-34a expression and suppressed proliferation of gastric cancer cells Cancer Biother Radiopharm 34: 634-641, 2019.

8. Qian C, Wang B, Zou Y, Zhang Y, Hu X, Sun W, Xiao H, Liu H and Shi L: MicroRNA 145 enhances chemosensitivity of glioblastoma stem cells to demethoxycurcumin. Cancer Manag Res 11: 6829-6840, 2019.

9. Sak K: Radiosensitizing potential of curcumin in different cancer models. Nutr Cancer 72: 1276-1289, 2020.

10. Xu R,LiH, Wu S, Qu J, Yuan H,Zhou Y and Lu Q: MicroRNA-1246 regulates the radio-sensitizing effect of curcumin in bladder cancer cells via activating P53. Int Urol Nephrol 51: 1771-1779, 2019.

11. Dou H, Shen R, Tao J, Huang L, Shi H, Chen H, Wang Y and Wang T: Curcumin suppresses the colon cancer proliferation by inhibiting $W n t / \beta$-catenin pathways via miR-130a. Front Pharmacol 8: 877, 2017

12. Liu J, Li M, Wang Y and Luo J: Curcumin sensitizes prostate cancer cells to radiation partly via epigenetic activation of miR-143 and miR-143 mediated autophagy inhibition. J Drug Target 25: 645-652, 2017.

13. Zhao W, Zhou X, Qi G and Guo Y: Curcumin suppressed the prostate cancer by inhibiting JNK pathways via epigenetic regulation. J Biochem Mol Toxicol 32: e22049, 2018.

14. Cao H, Yu H, Feng Y, Chen L and Liang F: Curcumin inhibits prostate cancer by targeting PGK1 in the FOXD3/miR-143 axis. Cancer Chemother Pharmacol 79: 985-994, 2017.

15. Liu T, Chi H, Chen J, Chen C, Huang Y, Xi H, Xue J and Si Y: Curcumin suppresses proliferation and in vitro invasion of human prostate cancer stem cells by ceRNA effect of miR-145 and lncRNA-ROR. Gene 631: 29-38, 2017.

16. Livak KJ and Schmittgen TD: Analysis of relative gene expression data using real-time quantitative PCR and the 2(-Delta Delta C(T)) method. Methods 25: 402-408, 2001.

17. Wu X, Jia R, Wang M, Chen S, Liu M, Zhu D, Zhao X, Yang Q, $\mathrm{Wu} \mathrm{Y}$, Yin Z, et al: Downregulation of microRNA-30a-5p contributes to the replication of duck enteritis virus by regulating Beclin-1-mediated autophagy. Virol J 16: 144, 2019.

18. Li J, Zhao LM, Zhang C, Li M, Gao B, Hu XH, Cao J and Wang GY: The lncRNA FEZF1-AS1 promotes the progression of colorectal cancer through regulating OTX1 and targeting miR-30a-5p. Oncol Res 28: 51-63, 2020.

19. Noori J, Haghjooy Javanmard S and Sharifi M: The role of microRNA-30a and downstream snaill on the growth and metastasis of melanoma tumor. Iran J Basic Med Sci 22: 534-540, 2019.

20. Zhao H, Lai X, Zhang W, Zhu H, Zhang S, Wu W, Wang S, Tang M, Deng Z and Tan J: MiR-30a-5p frequently downregulated in prostate cancer inhibits cell proliferation via targeting PCLAF. Artif Cells Nanomed Biotechnol 47: 278-289, 2019.

21. Yang J, Wang C, Zhang Z, Chen X, Jia Y, Wang B and Kong T: Curcumin inhibits the survival and metastasis of prostate cancer cells via the Notch-1 signaling pathway. APMIS 125: 134-140, 2017

22. Sha J, Li J, Wang W, Pan L, Cheng J, Li L, Zhao H and Lin W: Curcumin induces G0/G1 arrest and apoptosis in hormone independent prostate cancer DU-145 cells by down regulating Notch signaling. Biomed Pharmacother 84: 177-184, 2016.

23. Du Y, Long Q, Zhang L, Shi Y, Liu X, Li X, Guan B, Tian Y, Wang X, Li L, et al: Curcumin inhibits cancer-associated fibroblast-driven prostate cancer invasion through MAOA/ mTOR/HIF-1 $\alpha$ signaling. Int J Oncol 47: 2064-2072, 2015.

24. Sundram V, Chauhan SC, Ebeling M and Jaggi M: Curcumin attenuates $\beta$-catenin signaling in prostate cancer cells through activation of protein kinase D1. PLoS One 7: e35368, 2012

25. Wei Q, Tu Y, Zuo L, Zhao J, Chang Z, Zou Y and Qiu J: MiR-345-3p attenuates apoptosis and inflammation caused by oxidized low-density lipoprotein by targeting TRAF6 via TAK1/p38/NF-kB signaling in endothelial cells. Life Sci 241: $117142,2020$.

26. Wu Z, Chen D, Wang K, Cao C and Xu X: Long non-coding RNA SNHG12 functions as a competing endogenous RNA to regulate MDM4 expression by sponging miR-129-5p in clear cell renal cell carcinoma. Front Oncol 9: 1260, 2019.
27. Ye J, Lei J, Fang Q, Shen Y, Xia W, Hu X, Xu Q, Yuan H, Huang J and $\mathrm{Ni} \mathrm{C}$ : miR-4666-3p and miR-329 synergistically suppress the stemness of colorectal cancer cells via targeting TGF- $\beta / \mathrm{Smad}$ pathway. Front Oncol 9: 1251, 2019.

28. Zhang H, Li Y, Tan Y, Liu Q, Jiang S, Liu D, Chen Q and Zhang S: MiR-9-5p inhibits glioblastoma cells proliferation through directly targeting FOXP2 (Forkhead Box P2). Front Oncol 9: 1176, 2019.

29. Rui X, Gu TT, Pan HF, Shao SL and Shao HX: MicroRNA-381 suppresses proliferation and invasion of prostate cancer cells through downregulation of the androgen receptor. Oncol Lett 18: 2066-2072, 2019

30. Jiao DM, Yan L, Wang LS, Hu HZ, Tang XL, Chen J, Wang J, Li Y and Chen QY: Exploration of inhibitory mechanisms of curcumin in lung cancer metastasis using a miRNA- transcription factor-target gene network. PLoS One 12: e0172470, 2017.

31. Yin S, Du W, Wang F, Han B, Cui Y, Yang D, Chen H, Liu D, Liu X, Zhai X, et al: MicroRNA-326 sensitizes human glioblastoma cells to curcumin via the SHH/GLI1 signaling pathway. Cancer Biol Ther 19: 260-270, 2018.

32. Zhu X and Zhu R: Curcumin suppresses the progression of laryngeal squamous cell carcinoma through the upregulation of miR-145 and inhibition of the PI3K/Akt/mTOR pathway. OncoTargets Ther 11: 3521-3531, 2018.

33. Feng S, Wang Y, Zhang R, Yang G, Liang Z, Wang Z and Zhang G: Curcumin exerts its antitumor activity through regulation of $\mathrm{miR}-7 / \mathrm{Skp} 2 / \mathrm{p} 21$ in nasopharyngeal carcinoma cells. OncoTargets Ther 10: 2377-2388, 2017.

34. Zhang L, Zhang XW, Liu CH, Lu K, Huang YQ, Wang YD, Xing L, Zhang LJ, Liu N, Jiang H, et al: miRNA-30a functions as a tumor suppressor by downregulating cyclin E2 expression in castration-resistant prostate cancer. Mol Med Rep 14: 2077-2084, 2016.

35. Zhang J, Shen C, Wang L, Ma Q, Xia P, Qi M, Yang M and Han B: Metformin inhibits epithelial-mesenchymal transition in prostate cancer cells: Involvement of the tumor suppressor miR30a and its target gene SOX4. Biochem Biophys Res Commun 452: 746-752, 2014.

36. Jiang LH, Zhang HD and Tang JH: MiR-30a: A novel biomarker and potential therapeutic target for cancer. J Oncol 2018: 5167829, 2018.

37. Li L, Kang L, Zhao W, Feng Y, Liu W, Wang T, Mai H, Huang J, Chen S, Liang Y, et al: miR-30a-5p suppresses breast tumor growth and metastasis through inhibition of LDHA-mediated Warburg effect. Cancer Lett 400: 89-98, 2017.

38. Zhu J, Zeng Y, Li W, Qin H, Lei Z, Shen D, Gu D, Huang JA and Liu Z: CD73/NT5E is a target of miR-30a-5p and plays an important role in the pathogenesis of non-small cell lung cancer. Mol Cancer 16: 34, 2017.

39. Zhang T, Guo J, Gu J, Chen K, Wang Z, Li H, Wang G and Wang J: KIAA0101 is a novel transcriptional target of FoxM1 and is involved in the regulation of hepatocellular carcinoma microvascular invasion by regulating epithelial-mesenchymal transition. J Cancer 10: 3501-3516, 2019.

40. Abdelgawad IA, Radwan NH and Hassanein HR: KIAA0101 mRNA expression in the peripheral blood of hepatocellular carcinoma patients: Association with some clinicopathological features. Clin Biochem 49: 787-791, 2016.

41. Lv W, Su B, Li Y, Geng C and Chen N: KIAA0101 inhibition suppresses cell proliferation and cell cycle progression by promoting the interaction between p53 and Sp1 in breast cancer. Biochem Biophys Res Commun 503: 600-606, 2018.

42. Chen H, Xia B, Liu T, Lin M and Lou G: KIAA0101, a target gene of miR-429, enhances migration and chemoresistance of epithelial ovarian cancer cells. Cancer Cell Int 16: 74, 2016.

This work is licensed under a Creative Commons Attribution-NonCommercial-NoDerivatives 4.0 International (CC BY-NC-ND 4.0) License. 